\title{
Aircraft Trajectory Tracking with Large Sideslip Angles for Sense and Avoid Intruder State Estimation*
}

\author{
Peter Bauer ${ }^{1}$, Balint Vanek, Tamas Peni, Tamas Zsedrovits, Borbala Pencz, Akos Zarandy and Jozsef Bokor
}

\begin{abstract}
This paper deals with the problem of trajectory tracking and definition in monocular vision based sense and avoid scenarios for small UAVs where the intruder aerial vehicle should be held in camera field of view. After defining effective field of view (EFOV) and introducing its possible calculation tracking of a zig-zag trajectory by a conventional roll angle based tracker is examined. This results in zero EFOV and so probably impossible sensing and avoidance. In the next step the authors propose a new tracking solution which uses large sideslip angles to hold aircraft roll angle around zero. This way an acceptable EFOV can be provided. Further improvements in EFOV are gained through the definition of a sinusoidal trajectory which gives smoother tracking. All the results are demonstrated with software-in-the-loop simulation data.
\end{abstract}

\section{INTRODUCTION}

Sense and avoid (S\&A) capability is a crucial ability for the future unmanned aerial vehicles (UAVs). It is vital to integrate civilian and governmental UAVs into the common airspace according to [1]. In a S\&A scenario the own UAV is the observer which should estimate the direction and distance of the close other aircraft or UAV called intruder. The final goal is to estimate the probability of collision and initiate an avoidance maneuver if required.

Monocular vision based solutions can be cost and weight effective therefore especially good for small UAVs. However, their application needs persistent excitation of the filter which estimates the intruder distance and flying direction (see [2] for example). Several other sources point out that lateral acceleration of the observer is required to provide intruder observability ([3], [4], [5]).

In the previous work [2] a zig-zag (ZZ) trajectory defined by its corner points was used to give persistent excitation to the estimator. However, the tracking of this trajectory can cause to loose the intruder from camera field of view (FOV) because of rotation and translation of the aircraft. The solution could be the use of moving (rotating) camera or the decrease of rotations of the aircraft. For a cost and weight effective solution possibly the latter should be applied. This paper deals with the examination and solution of this problem including the following topics.

Section II deals with the definition and calculation of effective field of view (EFOV). Section III introduces the

IEEE ID of final version: 978-1-4799-5899-3/14/\$31.00 (C)2014 IEEE, published in proceedings of IEEE MED'14 pp. 1287-1292

*This work is supported by the Office of Naval Research Global, Grant Number N62909-10-1-7081, Dr. Paul Losiewicz program officer.

${ }^{1}$ All the authors are with Institute for Computer Science and Control, Hungarian Academy of Sciences (MTA SZTAKI), Budapest, Hungary peter.bauer@sztaki.mta.hu

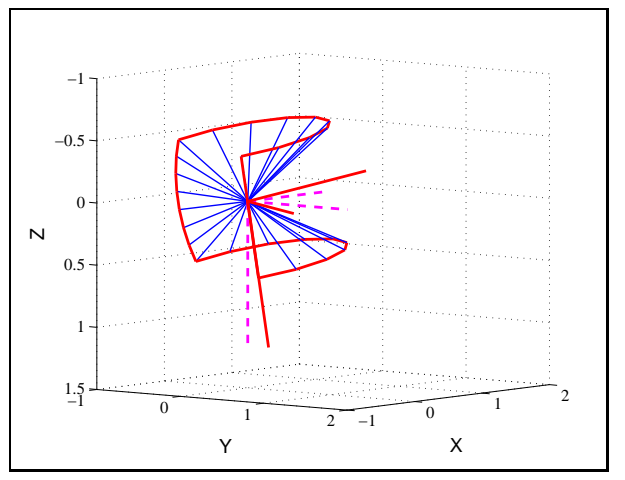

Fig. 1. Representation of camera FOV in camera (continuous) and NED (dashed) frames

trajectory tracking method originally applied to control the UAV which gives almost zero EFOV and then proposes a new solution which gives acceptable EFOV for the camera. Then section IV introduces the tracking of a sinusoidal trajectory instead of waypoints which further improves EFOV in combination with the newly developed tracking method. Finally section $\mathrm{V}$ concludes the paper.

\section{EFFECTIVE FIELD OF VIEW DEFINITION AND CALCULATION}

In the S\&A work we define camera FOV with the horizontal and vertical angle of views of the camera. In [6] a five-camera vision system was developed which has $220^{\circ}$ horizontal and $60^{\circ}$ vertical FOV.

In the representation of aircraft and camera motion the North-East-Down (NED) reference frame can be assumed to be an inertial frame during a S\&A task because the duration of such a task is only a few minutes. Aircraft motion can be described by the body, and camera motion by the camera coordinate frame (for details see [2] for example). In this work it is assumed that the camera frame is aligned with the body frame without loss of generality.

The camera FOV in camera frame can be represented as the 'rectangular hole' on a unit sphere as shown in Fig. 1 for the $220^{\circ} / 60^{\circ}$ FOV camera. This 'hole' can be represented by unit vectors describing its boundary on the sphere (see figure).

If one aligns the axes of the camera system with the NED frame (the camera points to the north) the FOV will be $\left[-110^{\circ}, 110^{\circ}\right]$ azimuth angle in the North-East plane and $\left[-30^{\circ}, 30^{\circ}\right]$ down and up in the North-Down plane. However, if one rotates or translates the camera the azimuth and up/down ranges will decrease on one side and increase on 
the other side. Considering a sequence of camera rotations and translations there will be a common $3 \mathrm{D}$ region in the NED frame which is narrower then the original FOV and is always observed by the camera. This way EFOV can be defined (it is defined from another point of view in [7]).

Definition 1: Effective field of view (EFOV): Consider a finite spatial trajectory of an aerial vehicle. Construct its camber line as the least squares optimal straight line fit. The effective field of view is the horizontal and vertical angle range relative to this line which is always in the onboard camera FOV.

Here it is assumed that the camber line of aerial vehicle trajectory is aligned with the North axis of NED frame (altitude hold controller is applied and the trajectory is symmetrical to the North axis). This way the azimuth and up/down angles in NED frame characterize the EFOV. The effect of rotation and translation on the EFOV is examined separately below.

\section{A. Effect of aircraft rotation on EFOV}

This effect can be examined considering the Euler angles and direction cosine matrix (DCM, transformation from earth to body (now also to camera) system) of the aircraft during trajectory tracking. Worst case (conservative) and less conservative methods can be used.

The worst case method selects the minimum and maximum roll $(\phi)$, pitch $(\theta)$ and yaw $(\psi)$ angles from the data and creates eight DCM-s from their possible combinations. Rotating the vector set which describes camera FOV in camera system with the inverse DCMs (camera to earth transformation) and projecting the rotated FOV into the NED frame gives the EFOV as the intersection of the projected FOVs. Mercator projection (see [8]) was used to transform the spherical surface to the East-Down plane but angles are plotted instead distances. The basic camera FOV with zero rotation was completely projected, from the rotated FOVs only the points in front of the East-Down plane (horizontal angle between $\left[-90^{\circ}, 90^{\circ}\right]$ ) were projected to make the figures more clear.

The less conservative method determines the convex hull of the three dimensional set of Euler angles and calculates the DCMs in the points of this hull (usually much more than eight points). The rotation and projection steps are the same. A larger EFOV is expected from this because the extremal $\phi, \theta$ and $\psi$ values rarely appear at the same time (as was assumed in the worst case method). Fig. 2 and 3 show the difference between the two methods. The intersection of the rectangular shapes gives EFOV. In Fig. 3 a larger negative vertical angle well below $-20^{\circ}$ can be observed in the EFOV. The detailed evaluation of EFOVs will be done later in the comparison of tracking methods.

\section{B. Effect of aircraft translation on EFOV}

This can be examined separately in the horizontal and vertical planes considering Fig. 4.

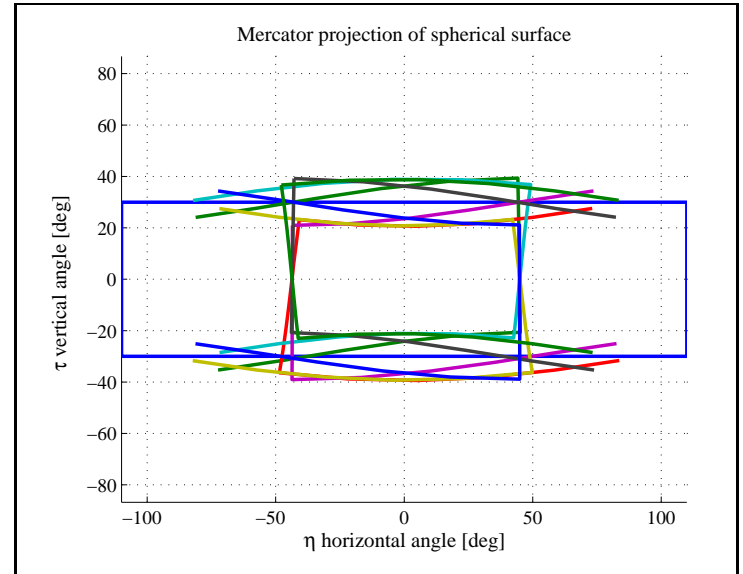

Fig. 2. EFOV with worst case method for ZZ trajectory tracking with proposed tracker

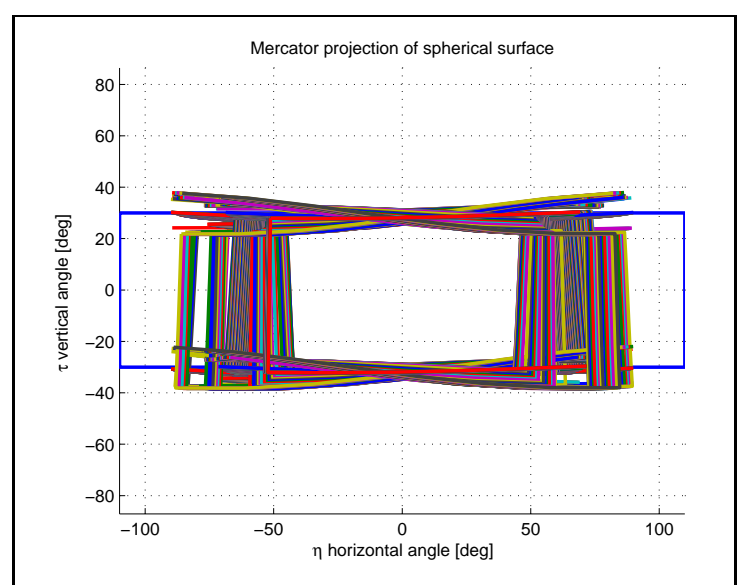

Fig. 3. EFOV with less conservative method for $\mathrm{ZZ}$ trajectory tracking with proposed tracker

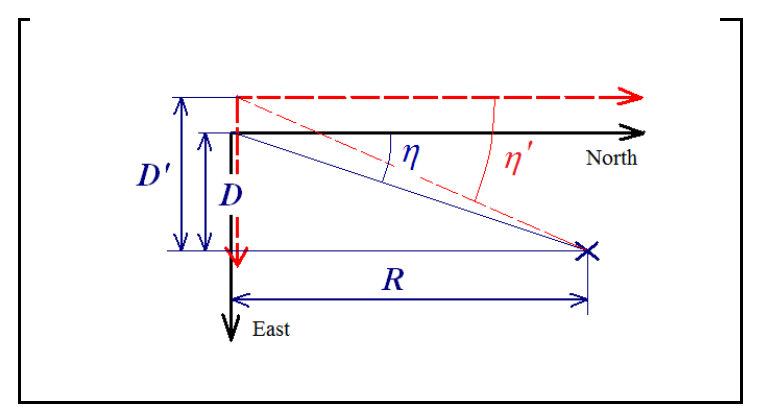

Fig. 4. Effect of aircraft translation on EFOV

The expressions are the same for the horizontal and vertical plane so the derivation is done only for one plane. The goal of the derivation is to characterize the change in the angle from $\beta$ to $\beta^{\prime}$ against the translational displacement of the aircraft. In Fig. 4 the forward distance of the intruder is $R$ the side distance is $D$ the angle of view is $\beta$. After a side displacement of the own aircraft with $\Delta D$ one gets $D^{\prime}=D+\Delta D$ side distance and a larger angle $\beta^{\prime}$.

The method of analysis is as follows: first consider the EFOV from aircraft rotation as in subsection II-A. The resulting angle can be considered as the larger angle $\beta^{\prime}$. From this and $\Delta D$ the smaller angle $\beta$ can be determined. This $\beta$ 
is the maximum angle in EFOV which guarantees that the intruder will be in camera FOV after the $\Delta D$ displacement. The calculations are summarized in (1). The examination can be done by plotting the resulting $\beta$ angles against $R$ for a given $\beta^{\prime}$ (from rotational EFOV) and $\Delta D$ (the maximum from aircraft trajectory). Plots will be included and analysed in the forthcoming sections.

$$
D=R \cdot \tan \beta^{\prime}-\Delta D, \quad \beta=\arctan (D / R)
$$

\section{TRAJECTORY TRACKING METHODS AND THEIR EFFECTS ON EFOV}

In this section the possibilities of tracking a zig-zag (ZZ) trajectory and their effect on EFOV will be examined. The algorithms are implemented on the E-flite Ultrastick $25 \mathrm{e}$ nonlinear software-in-the-loop simulation model developed in [9].

The ZZ trajectory is given by waypoints that's why the course angle based tracking algorithm published in [10] was applied. This calculates the reference course angle $\chi_{c}$ of the aircraft from A/C and waypoint (WP) north (X) / east (Y) position at every time as:

$$
\chi_{c}=\arctan 2\left(\frac{Y_{W P}-Y_{A / C}}{X_{W P}-X_{A / C}}\right)
$$

Originally the lateral control of the aircraft and so the trajectory tracking is implemented in the following way (call it as 'basic' method):

1) Track the trajectory by generating a roll angle reference from the course angle difference $\left(\phi_{\text {ref }}=K_{\phi} \cdot\left(\chi_{\text {ref }}-\right.\right.$ $\chi)$ where $\chi_{\text {ref }}$ reference, $\chi$ actual course angle and $K_{\phi}$ gain). $\phi_{\text {ref }}$ is tracked applying the aileron with PID control.

2) Turn coordination is applied using the rudder and PI control (this will hold the angle of sideslip around zero).

The strategy was tuned to track the waypoints as well as possible. This strategy results in large roll angles which decreases the EFOV to unacceptable levels, see Fig. 5. In the figure zero intersection of the rotated FOVs can be seen so the EFOV is zero in all directions. If we plot the EFOV with the less conservative method the correct evaluation of the figure is impossible (the figure is too crowded). Probably there is nonzero EFOV with $\pm 10^{\circ}$ limits which is unacceptably narrow. As a conclusion it can be stated that there is a large probability to lost the intruder from camera FOV which makes the sense and avoid task impossible. So some other tracking methods and/or trajectories should be designed.

Fig. 5 shows that the roll angle has the largest adverse effect on EFOV mainly because of the small vertical FOV of the camera. That's why a trajectory tracking strategy with small roll angles should be applied if possible. Such a strategy can be implemented with the following parts:

1) Track the trajectory by generating a yaw rate reference from the course angle difference $\left(r_{r e f}=K_{r} \cdot\left(\chi_{r e f}-\right.\right.$

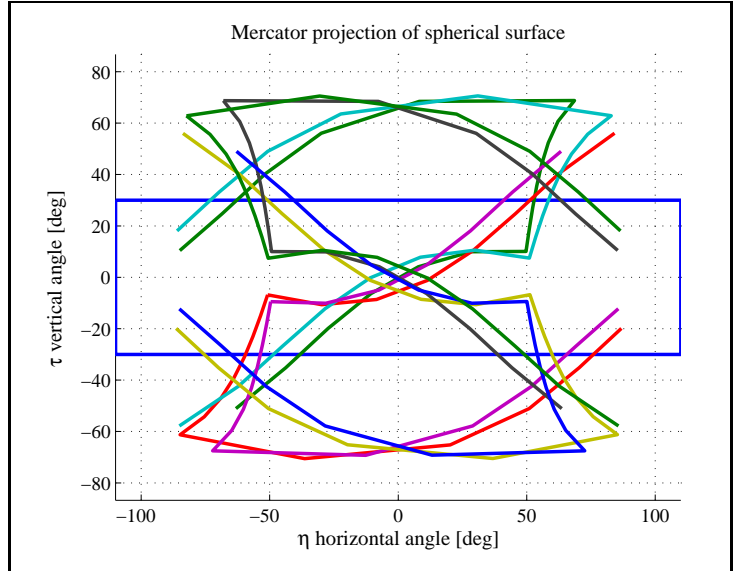

Fig. 5. EFOV with worst case method for ZZ trajectory tracking with basic tracker

$\chi)) . r_{r e f}$ is tracked applying the rudder with PI control (this will result in large angle of sideslip).

2) Attempt to hold zero roll angle using the aileron with PID control.

This method will be called 'proposed' in the forthcoming parts. It generates large angle of sideslip (AoS) which should be usually avoided by aircraft controllers. Large AoS means large side acceleration which could adversely effect a human pilot, but UAVs can well tolerate it. The method was again tuned to give the possible best tracking results. The EFOV with this method can be seen in Fig.-s 2 and 3. As the figures show there is a large and acceptable EFOV with the proposed tracking method.

The comparison of roll angles and the tracking results can be seen in Fig.-s 6 and 7. Tabular results are published in tables I and II.

TABLE I

ZZ TRAJECTORY TRACKING RESULTS basic (FIRST BLOCK), proposed (SECOND BLOCK)

\begin{tabular}{|c||c|c|c|c|c|c|}
\hline & $\phi$ & $\theta$ & $\psi$ & $\beta$ & $\Delta D_{y}$ & $\Delta D_{z}$ \\
\hline MIN & -40.4 & -5.38 & -56.8 & -3.48 & -62.7 & -5 \\
\hline MAX & 40.4 & 3.31 & 57.3 & 2.9 & 63.9 & 5 \\
\hline \hline MIN & -8.55 & -3.94 & -64.9 & -18.73 & -68.6 & -8 \\
\hline MAX & 8.4 & 2.94 & 66.2 & 19.4 & 65.9 & 8 \\
\hline
\end{tabular}

TABLE II

ZZ TRAJECTORY EFOV

\begin{tabular}{|c||c|c|c|c|}
\hline Method & $-\beta$ & $\beta$ & $-\alpha$ & $\alpha$ \\
\hline Worst case & -41.1 & 42.4 & -20.7 & 21 \\
\hline Less conservative & -42.5 & 43.8 & -26.4 & 21.8 \\
\hline
\end{tabular}

The tables show that the basic tracking gives smaller yaw but much larger roll angles (see also Fig. 6) than the proposed one. The smaller yaw angle can widen the EFOV, but this effect is destroyed by the large roll which makes the EFOV zero (see Fig. 5). The sideslip angles are much larger with the proposed method (see table I). The 
maximum horizontal $\left(\Delta D_{y}\right)$ and vertical $\left(\Delta D_{z}\right)$ translations are a bit larger with the proposed method (the tracking of the trajectory is a bit worse). However, this method gives an EFOV with about $\pm 42^{\circ}$ horizontal $(\beta)$ and $\pm 21^{\circ}$ vertical $(\alpha)$ range (the accurate data is in table II).

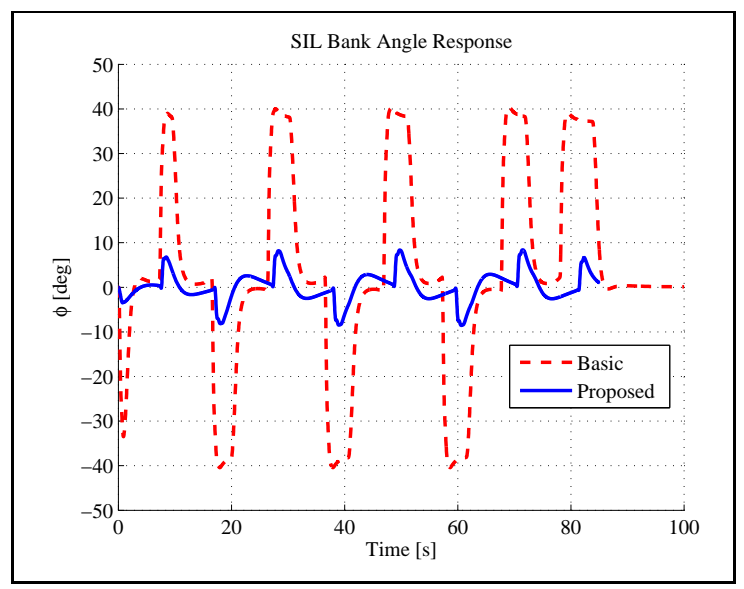

Fig. 6. Roll angle with basic and proposed trackers on ZZ trajectory

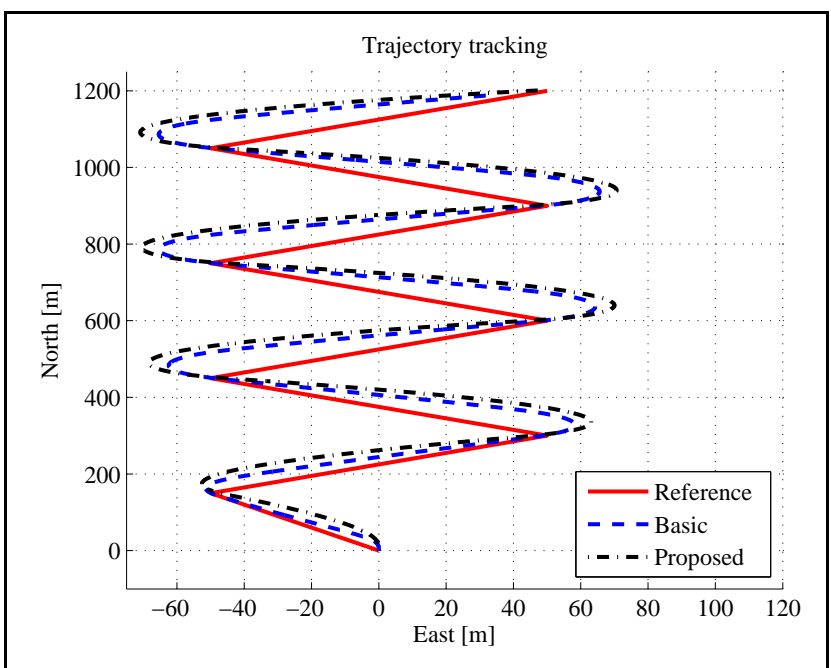

Fig. 7. Tracking results with basic and proposed trackers on ZZ trajectory

The effect of aircraft translation can be examined now considering $\pm 42^{\circ}$ horizontal and $\pm 21^{\circ}$ vertical angles (a rectangular and so conservative EFOV is considered for simplicity) and the maximum absolute horizontal $(69 \mathrm{~m})$ and vertical $(8 \mathrm{~m})$ displacements for an intruder distance $(\mathrm{R})$ from 0 to $3000 \mathrm{~m}$. The results can be seen in Fig. 8. The figure shows that the maximum allowed angle at observation of intruder is about constant for large $\mathrm{R}$ distances but then abruptly decreases by decreasing $\mathrm{R}$. There is always an $\mathrm{R}$ value where any small translation of the aircraft moves the intruder out from camera FOV. Considering a range of 500 to $3000 \mathrm{~m}$ for initial observation, the maximum allowed horizontal angles are $\pm 37.2^{\circ}$ and the verticals are $\pm 20.2^{\circ}$. This is the final EFOV of the given camera with the given $\mathrm{ZZ}$ trajectory and proposed tracker.

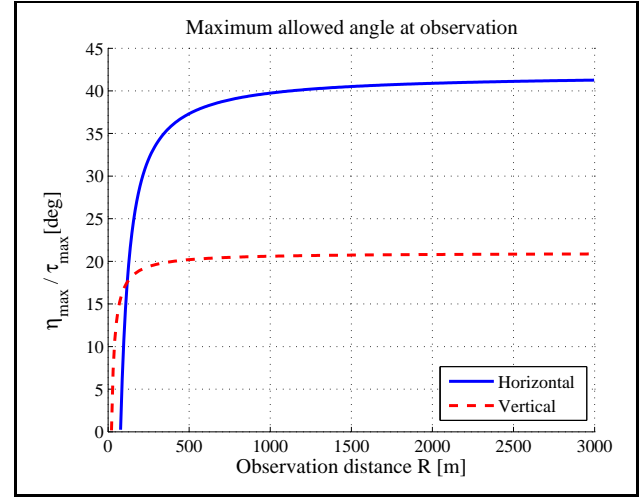

Fig. 8. Effect of aircraft translation on EFOV along ZZ trajectory

\section{SINUSOIDAL TRAJECTORY INSTEAD OF ZIG-ZAG ONE}

A possible further improvement can be to track a sinusoidal trajectory instead of the zig-zag one. This could give smoother and possibly smaller Euler angles and other data. Sinusoidal trajectory tracking was coded and flight tested for the Ultrastick aircraft (see [10]).

However, there is a problem with sinusoidal trajectory from S\&A point of view. The aircraft starts to follow the excitation trajectory ( $\mathrm{ZZ}$ or other) if it observes the intruder. At this point a relatively smooth transient from straight flight to the trajectory should be provided and the followed trajectory should be symmetrical to the original flight path. This way, the tracking of sinusoid should start at the zero crossing of the sine function, but this means a sudden change in aircraft direction and results in large Euler angles.

This problem was solved by the introduction of two transient waypoints which guide the aircraft to start the sinusoid at its negative peak, see Fig. 9. From the transient point the sinusoid is followed by the method introduced in [11] which is briefly summarized here.

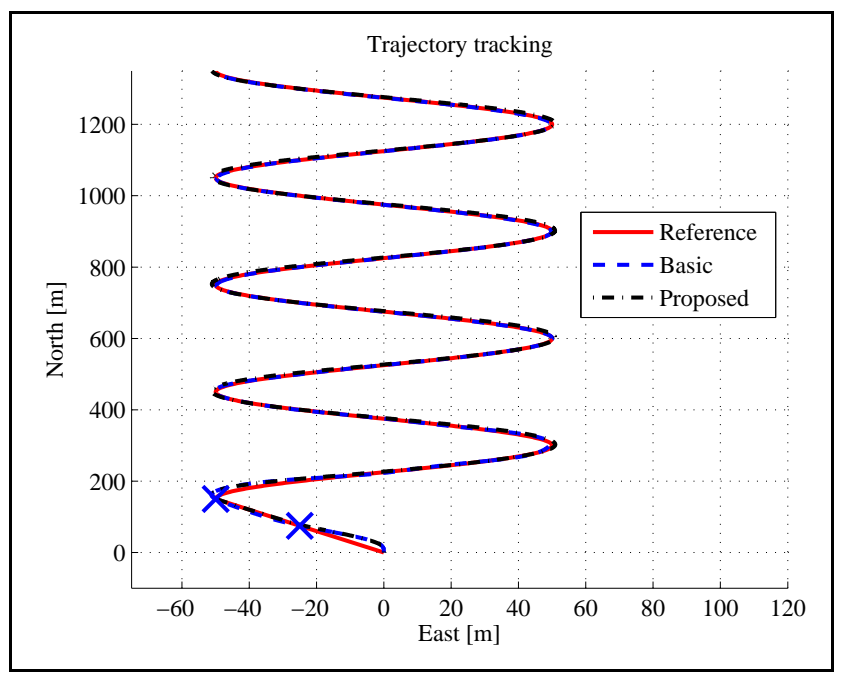

Fig. 9. Tracking results with basic and proposed trackers on SIN trajectory. The transient waypoints are also shown.

The sinusoid is given as a parametric trajectory: 
TABLE IV

SIN TRAJECTORY EFOV

$$
X=f_{x}(p)=p, \quad Y=f_{y}(p)=A \cdot \sin \left(\frac{2 \pi}{T} p\right)
$$

Here, $A$ is the amplitude (50m) $T$ is the period $(300 \mathrm{~m}$, the same as of the $\mathrm{ZZ}$ trajectory). Of course the whole sinusoid can be rotated to point in any direction in the North-East plane. The sinusoid is tracked by moving a virtual point on the trajectory $s$ meters forward of the A/C. In every step the $p$ parameter of this virtual point should be found. This can be done in multiple steps. First, move the actual point (parameter $p_{0}$ ) with the $\mathrm{A} / \mathrm{C}$ absolute ground velocity $(V)$ along the tangent of the trajectory ( $\Delta t$ time step):

$$
\begin{aligned}
& X_{V}=f_{x}\left(p_{0}\right)+\frac{\dot{f}_{x}\left(p_{0}\right)}{\sqrt{\dot{f}_{x}\left(p_{0}\right)^{2}+\dot{f}_{y}\left(p_{0}\right)^{2}}} V \Delta t \\
& Y_{V}=f_{y}\left(p_{0}\right)+\frac{\dot{f}_{y}\left(p_{0}\right)}{\sqrt{\dot{f}_{x}\left(p_{0}\right)^{2}+\dot{f}_{y}\left(p_{0}\right)^{2}}} V \Delta t
\end{aligned}
$$

Second, calculate the possible parameter of the new virtual point on the trajectory:

$$
p_{x}=f_{x}^{-1}\left(X_{V}\right) \quad p_{y}=f_{y}^{-1}\left(Y_{V}\right) \quad p(0)=\frac{p_{x}+p_{y}}{2}
$$

Finally, iterate the system of equations below with Newton-Raphson iteration for example to obtain the corrected $p_{0}=p$ parameter.

$$
\begin{aligned}
& \psi_{A}(0)=\arctan 2\left(\frac{f_{Y}(p(0))-Y_{A / C}}{f_{x}(p(0))-X_{A / C}}\right) \\
& \Phi\left(\psi_{A}, p\right)=\left[\begin{array}{c}
X_{A / C}+s \cdot \cos \left(\psi_{A}\right)-f_{x}(p) \\
Y_{A / C}+s \cdot \sin \left(\psi_{A}\right)-f_{y}(p)
\end{array}\right]=0
\end{aligned}
$$

Fig. 9 shows that the sinusoid (SIN) is followed better by both of the tracking methods (basic / proposed) and the tracking performances are almost equal. The EFOV with basic tracking is plotted in Fig. 10. It is again zero with the worst case and almost zero with the less conservative method.

The EFOV with the proposed tracking is plotted in Fig. 11 with the less conservative method. It is better than with the $\mathrm{ZZ}$ trajectory as the tables III and IV also show.

TABLE III

SIN TRAJECTORY TRACKING RESULTS basic (FIRST BLOCK), proposed (SECOND BLOCK)

\begin{tabular}{|c||c|c|c|c|c|c|}
\hline & $\phi$ & $\theta$ & $\psi$ & $\beta$ & $\Delta D_{y}$ & $\Delta D_{z}$ \\
\hline MIN & -40.7 & -5.24 & -48.2 & -2.88 & -51.4 & -5 \\
\hline MAX & 40.2 & 2.47 & 62.8 & 3.63 & 50.2 & 5 \\
\hline \hline MIN & -4.7 & -3.49 & -50.6 & -18.61 & -51.3 & -8 \\
\hline MAX & 7 & 1.94 & 54.5 & 16.58 & 51.1 & 8 \\
\hline
\end{tabular}

\begin{tabular}{|c||c|c|c|c|}
\hline Method & $-\beta$ & $\beta$ & $-\alpha$ & $\alpha$ \\
\hline Worst case & -53.2 & 56.9 & -22.2 & 22.7 \\
\hline Less conservative & -53.3 & 57.9 & -24.1 & 23.2 \\
\hline
\end{tabular}

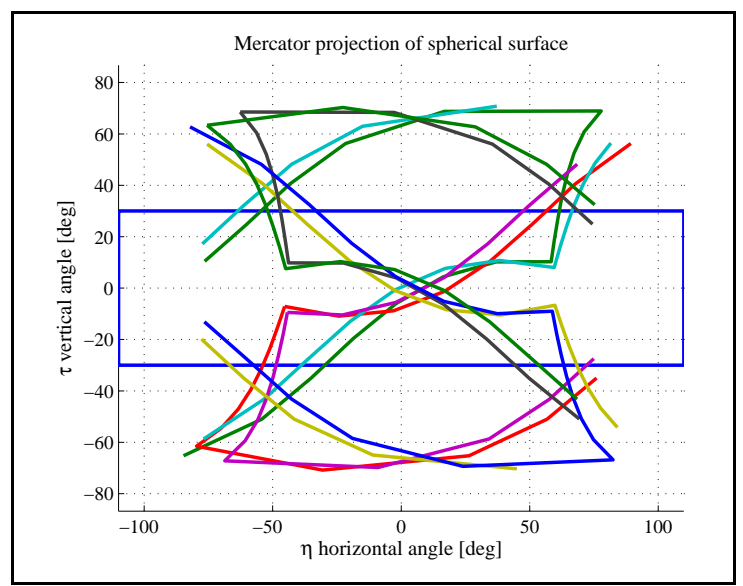

Fig. 10. EFOV with worst case method for SIN trajectory tracking with basic tracker

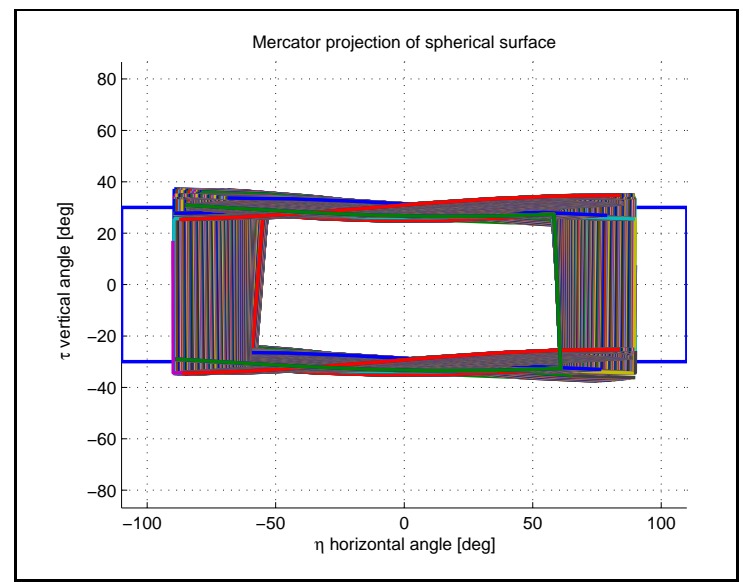

Fig. 11. EFOV with less conservative method for SIN trajectory tracking with proposed tracker

Table III shows that the roll and yaw angles are significantly smaller in following the sinusoid instead of the $\mathrm{ZZ}$ trajectory with the proposed tracker (compare to table I). The angle of sideslip is also a bit smaller for the sinusoid tracking. The horizontal translations are smaller both with basic and proposed trackers for the sinusoid.

In table IV the worst case and less conservative values are not as different as for the $\mathrm{ZZ}$ trajectory. The rectangular EFOV can be characterized by about $\pm 53^{\circ}$ horizontal and $\pm 23^{\circ}$ vertical range. The effect of translations is shown in Fig. 12 with $52 \mathrm{~m}$ horizontal and $8 \mathrm{~m}$ vertical displacements (from table III). The figure shows that the maximum allowed horizontal angles for initial observation are $\pm 50.7^{\circ}$ and the verticals are $\pm 22.2^{\circ}$ considering a range of 500 to $3000 \mathrm{~m}$. So, there is a large increase in horizontal EFOV and a small in vertical compared to results with ZZ trajectory. This is the 
final EFOV of the given camera with the given SIN trajectory and proposed tracker.

The comparison of roll angles can also be seen in Fig. 13.

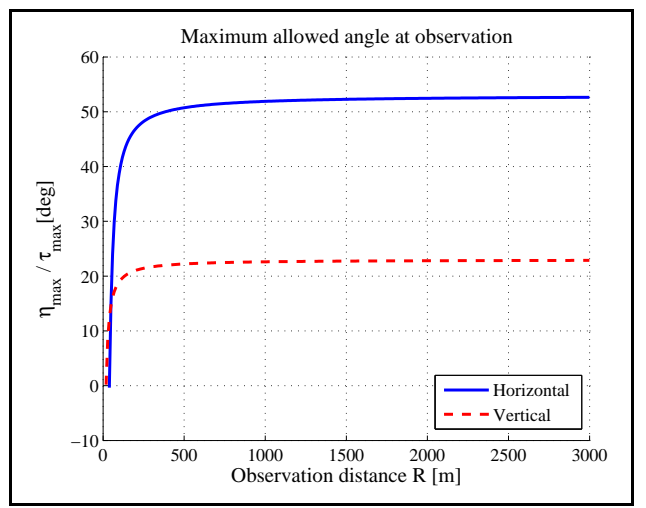

Fig. 12. Effect of aircraft translation on EFOV along SIN trajectory

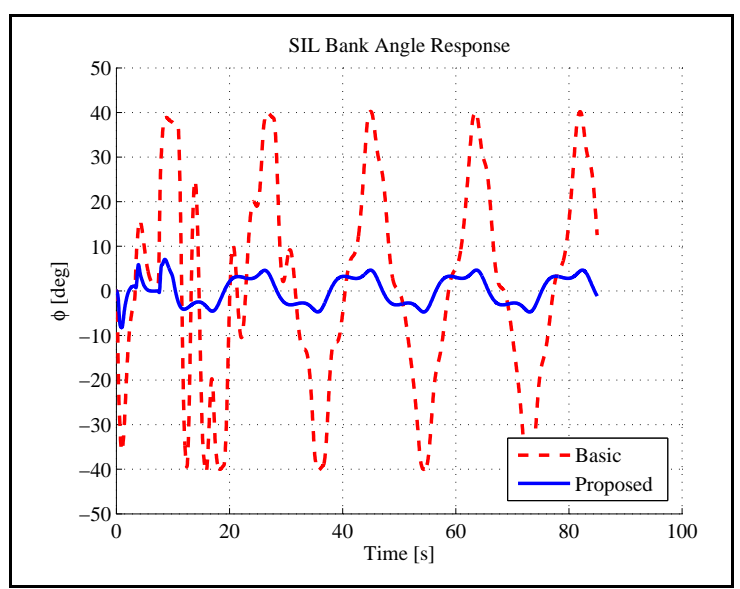

Fig. 13. Roll angle with basic and proposed trackers on SIN trajectory

\section{CONCLUSION}

This paper considers the problem of trajectory tracking and definition for sense and avoid intruder state estimation. A persistent excitation is required by the estimator which needs lateral acceleration of the aircraft. This excitation can be provided by following zig-zag like trajectories. However, the following of such trajectories with the conventional roll angle based tracking algorithms (see [10] for example) can possibly result in loss of intruder from camera field of view (FOV).

The paper first defines the expression of effective field of view (EFOV) and describes some possible methods to calculate it from aircraft rotation and translation.

Then these methods are applied to examine EFOV with a simulated zig-zag (ZZ) trajectory followed with a basic roll angle based tracker in software-in-the-loop (SIL) simulation of a real UAV. The results show that there will be almost no EFOV, so the intruder will possibly be lost from camera FOV which can make the sense and avoid task impossible.

That's why a new tracking method is introduced with which an acceptable EFOV can be gained (call it 'proposed'). This tracker is unconventional because it uses large sideslip angles to hold almost zero roll and so effectively increase EFOV. This is a feasible solution for UAVs where there is no pilot onboard and so no difficulty with large side acceleration.

Another possible development is the redefinition of aircraft trajectory to be a smooth curve instead of the $\mathrm{ZZ}$ waypoints. A sinusoidal trajectory can generate the same lateral accelerations but with smoother transients and so smaller Euler angles. The increase in EFOV by tracking the sinusoidal path with the proposed method is demonstrated through SIL simulation again. This configuration has superior performance over the others.

The topic of future work will be the validation of methods in hardware-in-the-loop simulations including image generation and processing, intruder state estimation and collision probability calculation (see [12]). The effect of wind disturbances in the tracking will be also considered.

Real flight tests with the proposed controller will also be conducted.

\section{REFERENCES}

[1] "Roadmap for the integration of civil Remotely-Piloted Aircraft Systems into the European Aviation System," European RPAS Steering Group, Tech. Rep., 2013.

[2] B. Vanek, T. Peni, A. Zarandy, J. Bokor, T. Zsedrovits, and T. Roska, "Performance Characteristics of a Complete Vision Only Sense and Avoid System," in in Proceedings of AIAA GNC 2012 (Guidance, Navigation and Control Conference), no. AIAA 2012-4703, Minneapolis, Minnesota, August 2012, pp. 1-15.

[3] Y. Watanabe, "Stochastically Optimized Monocular Vision-based Navigation and Guidance," Ph.D. dissertation, Georgia Institute of Technology, 2008.

[4] L. Matthies, R. Szeliski, and T. Kanade, "Kalman Filter-based Algorithms for Estimating Depth from Image Sequences," Carnegie-Mellon University, Tech. Rep. CMU-CS-87-185, 1987.

[5] S. S. Ponda, R. M. Kolacinski, and E. Frazzoli, "Trajectory Optimization for Target Localization Using Small Unmanned Aerial Vehicles," in In proceedings of AIAA GNC 2009 (Guidance Navigation and Control Conference), no. AIAA 2009-6015. Chicago, Illinois, USA: AIAA, August 2009.

[6] A. Zarandy, Z. Nagy, B. Vanek, T. Zsedrovits, A. Kiss, and M. Nemeth, "A Five-Camera Vision System for UAV Visual Attitude Calculation and Collision Warning," in Computer Vision Systems, Lecture Notes in Computer Science, Saint Petersburg, Russia, July 2013, pp. 11-20.

[7] F. Z. Brill, G. S. Wasson, G. J. Ferrer, and W. N. Martin, "The effective field of view paradigm: adding representation to a reactive system," Engineering Applications of Artificial Intelligence, vol. 11, no. 2, pp. 189-201, 1998.

[8] J. C. Polking. (2000) Mapping the sphere. PDF document. [Online]. Available: http://math.rice.edu/ polking/cartography/cart.pdf

[9] Y. C. Paw, "Synthesis and Validation of Flight Control for UAV," $\mathrm{Ph} . \mathrm{D}$. dissertation, University of Minnesota, 2009.

[10] P. Bauer and A. Dorobantu, "Optimal Waypoint Guidance, Trajectory Design and Tracking," in In proceedings of American Control Conference 2013. Washington DC, USA: AACC, 2013.

[11] P. Bauer and J. Bokor, "Tuning and Improvements in a Waypoint and Trajectory Tracking Algorithm," in Proceedings of AIAA Guidance, Navigation and Control Conference, no. AIAA 2012-4604. Minneapolis, MN., USA: AIAA, 13-16 August 2012.

[12] T. Zsedrovits, A. Zarandy, B. Vanek, T. Peni, J. Bokor, and T. Roska, "Collision avoidance for UAV using visual detection," in in Proceedings of IEEE ISCAS 2011 (International Symposium on Circuits and Systems). Rio de Janeiro: IEEE, May 2011, pp. 2173-2176. 